\title{
Isolation and Identification of a New Strain of Nervous Necrosis Virus from the Big-belly Seahorse Hippocampus Abdominalis in China
}

\section{Xinxin Chen}

Xiamen Xiaodeng Fisheries and Technology Co., LTD https://orcid.org/0000-0002-6225-0844

Jianfei Qi

Fisheries Research Institute of Fujian

Libin He

Fisheries Research Institute of Fujian

Huiyu Luo

Fisheries Research Institute of Fujian

Jinbo Lin

Xiamen Xiaodeng Fisheries Science and Technology Co., LTD

\section{Fengyan Qiu}

Xiamen Xiaodeng Fisheries Science and Technology Co., LTD

\section{Qing Wang}

South China Agricultural University College of Marine Sciences

Leyun Zheng ( $D$ 981668817@qq.com)

Fisheries Research Institute of Fujian

\section{Research}

Keywords: Nervous necrosis virus, Big belly seahorse, Identification, Pathogenicity

Posted Date: November 1st, 2021

DOI: https://doi.org/10.21203/rs.3.rs-970769/v1

License: (1) (1) This work is licensed under a Creative Commons Attribution 4.0 International License.

Read Full License 


\title{
Isolation and identification of a new strain of nervous necrosis virus from the big-belly seahorse Hippocampus abdominalis in China
}

\author{
Xinxin Chen ${ }^{1, \dagger}$, Jianfei $\mathrm{Qi}^{2, \dagger}$, Libin $\mathrm{He}^{2}$, Huiyu Luo ${ }^{2}$, Jinbo Lin ${ }^{1}$, Fengyan Qiu ${ }^{1}$, \\ Qing Wang ${ }^{3,4, *}$, Leyun Zheng ${ }^{2, *}$
}

*Correspondence: wangqing@scau.edu.cn and 981668817@qq.com.

${ }^{\dagger}$ Xinxin Chen and Jianfei Qi have contributed equally to this work.

\author{
Author details \\ ${ }^{1}$ Xiamen Xiaodeng Fisheries Science and Technology Co., LTD, Xiamen, 361006, China. \\ ${ }^{2}$ Fisheries Research Institute of Fujian, Xiamen, 361000, China. \\ ${ }^{3}$ College of Marine Sciences, South China Agricultural University, Guangzhou 510642, China. \\ ${ }^{4}$ Guangdong Laboratory for Lingnan Modern Agriculture, Guangzhou, 510642, China.
}




\section{Abstract}

Background: Betanodaviruses, members of the Nodaviridae family, are the causative agents of viral nervous necrosis (VNN) in fish, resulting in great economic losses worldwide.

Methods: In this study, we isolated a virus strain named seahorse nervous necrosis virus (SHNNV) from cultured big-belly seahorses Hippocampus abdominalis in Xiamen city, Fujian Province, China. Cell isolation, PCR detection, phylogenetic analysis, qRT-PCR, fluorescence in situ hybridization (FISH) and histology were used for virus identification and analysis of virus histopathology. Furthermore, an artificial infection experiment was conducted for virulence testing.

Results: Brain and eye tissue homogenates of diseased big-belly seahorses were inoculated onto a grouper spleen (GS) cell monolayer at $28^{\circ} \mathrm{C}$. Tissue homogenates induced obvious cytopathic effects in GS cells. PCR and sequencing analyses revealed that the virus belonged to Betanodavirus and shared high sequence identity with red-spotted grouper nervous necrosis virus (RGNNV) isolates. qRT-PCR and fluorescence in situ hybridization revealed that SHNNV mainly attacked the brain and eye. Histopathological examination revealed that the virus led to cytoplasmic vacuolation in the brain and retinal tissues. Infection experiments confirmed that SHNNV was highly infectious, causing massive death in big-belly seahorses.

Conclusion: A novel seahorse betanodavirus from the big-belly seahorse in China was discovered. This finding will contribute to the development of efficient strategies for disease management in aquaculture.

Keywords: Nervous necrosis virus, Big-belly seahorse, Identification, Pathogenicity 


\section{Background}

Seahorses are a group of aquatic animals that have been used for many years for their medicinal and ornamental properties. Annually, tens of millions of seahorses are collected from the wild and enter trade globally, mostly used for medicinal purposes [1-4]. Being scarce in population, seahorses are listed in the International Union for Conservation of Nature (IUCN) Red List of Threatened Species and the Convention on International Trade in Endangered Species of Wild Fauna and Flora (CITES) [5,6]. The big-belly seahorse (Hippocampus abdominalis) is one of the largest seahorse species, and is mostly distributed in the temperate coastal regions of New Zealand and south-eastern Australia [6]. Owing to its high market value, the big-belly seahorse has become one of the major mariculture species in China in recent years, although full of challenges. One of the major threats to seahorse cultivation is frequent outbreaks of viral diseases, causing heavy economic losses.

Nervous necrosis viruses (NNVs) are non-enveloped positive-sense RNA viruses classified in the family Nodaviridae, which contains two genera: betanodaviruses, which predominantly infect fish, and alphanodaviruses, which predominantly infect insects [7]. Betanodaviruses-infected fish show abnormal swimming behaviour, such as spiraling and darting, due to vacuolation and necrosis of the central nervous system [8], furthermore, NNVs result in high mortality in hatchery-reared larvae and juveniles of a wide range of marine fish species in Asia, Europe, Australia, North America and North Africa [9-16]. The genome of NNV, packed into a diameter of 25-30 nm icosahedral virion, is comprised of two positive-sense RNA molecules: RNA1 encodes the RNA-dependent RNA polymerase (RdRp) required for viral genome replication, and RNA2 encodes the capsid protein $(\mathrm{Cp})$, which is the single major structural protein of NNV and determines the host specificity $[9,17,18]$. According to the International Committee on Taxonomy of Viruses, NNVs are classified into four genotypes: striped jack NNV (SJNNV), tiger puffer NNV (TPNNV), barfin flounder NNV (BFNNV), and red-spotted grouper NNV (RGNNV) [18]. Highly contagious and virulent, NNV of all genotypes has been identified in more than 40 species of marine and freshwater fish, either asymptomatic or causing neural disease $[9,19]$. However, no NNV infected seahorses have been recorded.

Recently, we have observed a disease causing spiral swim pattern, abdominal distension and high lethality in farmed seahorses in Fujian, China, without parasitic infection, bacterial infection, mycotic infection, external or organ injuries or water pollution. It was highly suspected that the seahorses were infected with viruses, and we analysed the pathogens in this work. Our study is the first identification of a virus causing VNN in seahorses, and we designate it seahorse NNV (SHNNV). We report the isolation, characterization and pathogenicity of a SHNNV isolate from the big-belly seahorse. The present study provides a basic resource for the subsequent investigation of big-belly seahorse and SHNNV interactions, as well as a new applicable species for NNV research. 


\section{Methods}

\section{Sample collection}

In this study, diseased big-belly seahorses (average weight of $0.33 \pm 0.12 \mathrm{~g}$, average body length of $7.21 \pm 1.03 \mathrm{~cm}$ ) were obtained from a fish farm in Xiamen city, Fujian Province, China. The fish showed abnormal swimming behaviour, and the cumulative mortality rate was approximately $40 \%$ in one month. Eye, brain, rhynchodaeum, liver, intestine, brood pouch, bone, muscle, gonad, heart, kidney, gill, skin and gallbladder tissues of each fish were analysed individually for the presence of NNVs.

\section{Viral isolation}

Eye and brain tissue samples of NNV-positive seahorses were homogenized in $5 \mathrm{~mL}$ of L15 medium (Gibco, USA) without foetal bovine serum (FBS). Then, the tissue homogenates were filtered through a $0.22 \mu \mathrm{m}$ filter membrane, inoculated on grouper spleen (GS) cells, and cultured in L15 medium supplemented with 10\% FBS (Gibco, USA). The same volume of L15 was added to the mock infected group. Inoculated cells were cultured at $28^{\circ} \mathrm{C}$ and monitored regularly for the development of cytopathic effects (CPEs). The virus isolate was propagated in GS cells until the cell monolayer was destroyed. The cell culture supernatant was then recovered, centrifuged at $1,000 \times \mathrm{g}$ for $10 \mathrm{~min}$ at $4^{\circ} \mathrm{C}$ and stored at $-80^{\circ} \mathrm{C}$ until use. Viral titres were determined by the $50 \%$ tissue culture infective dose (TCID50) method.

\section{Identification of the isolated viruses by PCR}

Total RNA was extracted from the supernatants of CPE-positive cultures using an RNA extraction kit (Takara, Japan) and reverse transcribed using a Transcription First Strand cDNA Synthesis Kit (Roche, Switzerland) according to the manufacturer's instructions. For the detection of SHNNV, PCR was performed using Blend Taq DNA polymerase (Toyobo, Japan) with the following conditions: denaturation at $94{ }^{\circ} \mathrm{C}$ for $2 \mathrm{~min}$, followed by 35 cycles of $94{ }^{\circ} \mathrm{C}$ for $30 \mathrm{~s}, 55^{\circ} \mathrm{C}$ for 30 $\mathrm{s}$, and $72{ }^{\circ} \mathrm{C}$ for $90 \mathrm{~s}$. The reactions were completed with a final extension of $10 \mathrm{~min}$ at $72{ }^{\circ} \mathrm{C}$. The PCR product was analysed by electrophoresis on a $1.5 \%$ agarose gel in TAE buffer containing GoldView I nucleic acid stain (Solarbio, China). The primers used are listed in Table 1. PCR products were cloned into PMD-18Tvectors (Takara, Japan) and sequenced by a sequencing company (BGI, China).

\section{Sequence alignment analysis}

The putative amino acid sequences were predicted using BioEdit software [20]. Phylogenetic analysis based on the protein sequence was performed with MEGA 6.0 using the neighbour joining method with resampling with 1000 bootstrap replicates [21].

\section{Tissue distribution of viruses}

To investigate which tissues the virus mainly infects, total RNA was extracted from different 
tissues of diseased big-belly seahorses, including the eye, brain, rhynchodaeum, liver, intestines, brood pouch, bone, muscle, gonad, heart, kidney, gill, skin and gallbladder. Transcripts of $c p$ gene from different tissues were examined by quantitative real-time PCR (qRT-PCR). The qRT-PCR conditions were as follows: denaturation at $94^{\circ} \mathrm{C}$ for $1 \mathrm{~min}$, followed by 40 cycles of $94^{\circ} \mathrm{C}$ for $15 \mathrm{~s}$, $55^{\circ} \mathrm{C}$ for $15 \mathrm{~s}$, and $72^{\circ} \mathrm{C}$ for $60 \mathrm{~s}$. Standard amplification curves for different genes were generated via serial dilutions of plasmid constructs. The concentration of the template in the samples was determined by relating the $\mathrm{Cq}$ value to the standard curve. The $\beta$-actin gene was used as an internal control. The primers used in this study are listed in Table 1 .

\section{Fluorescence in situ hybridization (FISH)}

Sense and antisense digoxigenin (DIG)-labelled riboprobes were synthesized from the open reading frame sequence of the $c p$ gene using a DIG RNA Labelling Kit (Roche Diagnostics, Germany).

The procedures for RNA FISH followed those of Ragoczy et al [22], Ho et al [23], and Beliveau et al [24], with modifications. Briefly, NNV-positive seahorses were fixed in buffered $4 \%$ paraformaldehyde for $24 \mathrm{~h}$. The samples were then dehydrated with a series of graded ethanol solutions (70\%-100\%), cleared in xylene and embedded in paraffin. Ten-micron sections were cut for FISH. Prior to hybridization, the slides were washed with phosphate-buffered saline (PBS), sequentially dehydrated in $70 \%, 90 \%$, and $100 \%$ ethanol, and equilibrated in $10 \%$ formamide / $2 \times$ saline sodium citrate(SSC), $\mathrm{pH}$ 7.0. A mixture of the primary sense and antisense probes and the secondary probes was hybridized to the cells in $10 \%$ formamide / $10 \%$ dextran sulfate / $2 \times \mathrm{SSC}$ / $5 \mathrm{mmol} / \mathrm{L}$ ribonucleotide vanadate complex / $0.05 \%$ bovine serum albumin / $1 \mu \mathrm{g} / \mu \mathrm{L} E$. coli tRNA and hybridized overnight at $55{ }^{\circ} \mathrm{C}$ in a humidified chamber. Slides were sequentially washed in $10 \%$ formamide / $2 \times \mathrm{SSC}$, pH 7.0, followed by $2 \times \mathrm{SSC}$ at $37{ }^{\circ} \mathrm{C}$ and then mounted with Fluoroshield with DAPI (Sigma, USA). Fluorescence signals from FISH were imaged with a Zeiss confocal microscope (Germany).

\section{Brain and eye histology}

The brains and eyes were removed from diseased and healthy big-belly seahorses, fixed in Bouin's solution overnight at room temperature, dehydrated, and then embedded in paraffin wax. All tissue blocks were sectioned at a thickness of $5 \mu \mathrm{m}$ and stained with hematoxylin and eosin for subsequent analysis.

\section{Experimental infection}

To investigate the pathogenicity of SHNNV, juvenile big-belly seahorses were experimentally infected with SHNNV. Juvenile big-belly seahorses (average body length of $7.89 \pm 0.40 \mathrm{~cm}$ ) were purchased from a fish farm and maintained at $21 \pm 1^{\circ} \mathrm{C}$ in tanks under a natural photoperiod. Juvenile big-belly seahorses were divided into two groups with 50 fish per group. A viral suspension was diluted tenfold, and each fish in the infection group received $30 \mu \mathrm{L}$ diluted virus by intraperitoneal injection. The control group was injected with $30 \mu \mathrm{L}$ PBS per seahorse. Seahorses were monitored twice daily for clinical signs of pathology and mortality. 


\section{Statistical analysis}

The results were analysed statistically using repeated measurements of variance analysis with SPSS software 25 [25].

\section{Results}

\section{Isolation and identification}

Brain and eye tissue homogenates of diseased big-belly seahorses were inoculated onto a GS cell monolayer at $28^{\circ} \mathrm{C}$. Significant CPEs were observed at $24 \mathrm{~h}$ in GS cells infected with virus (Figure 1B). No CPE was observed in the control group (Figure 1A). The infected cells were NNV-positive by PCR assays, and the PCR products of the estimated size (1073 bp) were identified, with clear electrophoretic bands (Figure 2). The $1017 \mathrm{bp}$ full-length gene encoded 338 amino acids (Sequence S1 and S2). A phylogenetic tree was constructed by the neighbour-joining method with1000 bootstrap replicates based on a multiple alignment of the gene sequences, which were predicted using the standard genetic code. The tree showed that the virus clustered with $\mathrm{NNV}$ and was most closely related to RGNNV; therefore, the isolated virus strain was designated as SHNNV (Figure 3).

\section{Tissues mainly infected with SHNNV}

To investigate the target tissues of SHNNV, we examined the transcription level of $c p$ mRNA in various tissues from diseased big-belly seahorses by qRT-PCR. As shown in Figure 4, its transcriptional level was significantly increased in the brain and eye. The virus was also found in brood pouch, skin, muscle and other organs, but only with extremely low viral load. We further performed FISH of the $c p$ mRNA. Positive signals were also detected in the brain and retina (Figure 5). These results indicate that SHNNV mainly infects the brain and eye of big-belly seahorses.

\section{Histopathology of SHNNV infection}

To investigate the pathology of big-belly seahorse brain and retinal tissue caused by SHNNV infection, we performed histological analysis on brain and retinal tissues of healthy and diseased seahorses. Compared to the healthy ones, SHNNV-positive seahorses showed cytoplasmic vacuolation in the brain (Figure $6 \mathrm{~A}$ and $\mathrm{B}$ ) and retinal tissues (Figure $6 \mathrm{C}$ and D).

\section{SHNNV isolate pathogenicity tests}

To evaluate the pathogenic potential of SHNNV in fish hosts, big-belly seahorses were infected 
with the virus by intraperitoneal injection. In the infection group, the seahorses began to die from the second day, and the survivors tended to stabilize over the ten days. The death rate was $92 \%$. In the control group, only two seahorses died, with a mortality rate of $4 \%$ (Figure 7).

\section{Discussion}

In the present study, a virus strain was isolated from diseased big-belly seahorses in Xiamen city. By employing a series of biochemical and biophysical methods, we first identified that the causative agent was an SHNNV strain belonging to betanodavirus. The history of VNN researches can be traced back to the mid-1980s. In 1985, a new disease with a mortality rate greater than $90 \%$ occurred in Australia, Japan, Southeast Asia, and the Caribbean in almost simultaneous outbreaks in the breeding of a variety of young marine fish [10,13,26,27]. In China, the first NNV isolation was reported in reared stone red-spotted grouper in 2001 [28]. Although some cases of similar symptoms have been discovered in numerous marine fish infected with NVV, no formal reports on seahorse nervous necrosis have been recorded. Thus, the first observation of a disease causing swimming in spirals or rotations, lethargy and anorexia in seahorses has not been reminiscent of $\mathrm{NNV}$ at the beginning, whereas further environmental and pathogenic analysis indicated virus infection. The typical symptom of VNN and the afterwards demonstrated vacuolation in the brain and eye confirmed that the causative agent belonged to betanodavirus. The pathogenic test showed acute viral infection and an extremely high case fatality rate of $92 \%$ compared to the control group (4\%).

In previous studies, researchers commonly identified and classified NNV by analysing the sequences of RNA2 or the $c p$ genes from various viral isolates [7,14,29,30]. $C p$ gene encodes the capsid protein, which contains a conservative shell domain (S-domain), a hypervariable protrusion domain (P-domain) and an N-terminal arm recruiting genomic RNA [31]. It has been indicated that $\mathrm{CP}$ is the major determinant of immunoactivity and host specificity [17,32], and partially determines the thermotolerance of NNV $[33,34]$. Here we identified the virus by analysing the full length of $c p$ mRNA amplified by primers that designed according to a known NNV isolate. Phylogenetic analysis indicated that SHNNV was most closely related to RGNNV genotype.

Previous studies have shown that RGNNV mainly destroys the brain and eyes of the infected fish, and the prominent pathological features are vacuolation of the brain and retina, resulting in massive losses to the population of grouper and other marine fishes [8,35,36]. It is reported that RGNNV also detected in gills, intestine, stomach, spleen, liver, kidney, pyloric gland, skeletal muscle, blood cells and fin $[8,9,36]$. In the present study, according to the results of the tissue distribution of the virus, brain and eye histology and FISH, we found that SHNNV mainly destroyed the big-belly seahorse brain and eye and caused vacuolation of the brain and retina. These results were consistent with the pathology of NNVs. We also detected a low expression level of viral mRNA in rhynchodaeum, liver, intestine, brood pouch, bone, muscle, gonad, heart, kidney, gill, skin and gallbladder tissues. 
In our unpublished study, we found that SHNNV was sensitive to water temperature. Many studies have discovered a temperature-dependent effect of NNV infection, with distinct optimum growth temperature in four genotypes. Unlike the other three genotypes that replicate in cold water, the optimum temperature for RGNNV replication was $25-28^{\circ} \mathrm{C}$, corresponding to the water temperature of VNN outbreaks [34,37-41]. The temperature range for RGNNV genotype, however, has not yet been definitely verified, while temperature that too high or too low was unsuitable for host cells to grow[42]. In our study, the seahorses were raised at $21^{\circ} \mathrm{C}$, and our further research showed that SHNNV could replicate at $13^{\circ} \mathrm{C}$, the temperature was far below the temperature optima, and below the lowest experimental temperature record [33,42,43]. The seahorse Hippocampus abdominalis can be cultured as low as $8^{\circ} \mathrm{C}$, and compared to other fish, it is small, rapid-propagative and easy to culture in experimental conditions, giving it an advantage for NNV researches.

A research showed a distinct difference between the RNA2 sequences of RGNNV isolates in fish held at high temperature and low temperature [44]. However, it is also reported that RNA1, encoding RdRp, protein B1 and B2, is the key contributing factor for the response to temperature [33], Ciulli et al. also considered that RNA1 rather than RNA 2 plays a more important part in thermotolerance of NNV, although RNA2 was found to determine temperature sensitivity as well [34,43]. There is evidence that the RNA1 evolved rapidly than RNA2, indicating a strong selection to respond to various temperature ranges $[15,45,46]$. In our study, we only analysed the $c p$ gene but not the complete sequence of RNA1 and RNA2. The next plan of analysing the genetic divergence of between SHNNV and other RGNNV isolates will be supplement to the studies of the thermal adaptability of NNV.

\title{
Conclusions
}

In conclusion, we isolated and characterized SHNNV from reared big-belly seahorses in China. The present isolate was closely related to known RGNNV isolates. It can infect GS cells and induce obvious CPE. We also found that SHNNV mainly destructs the big-belly seahorse brain and eye and causes vacuolation of the brain and retina. The infection experiment revealed that SHNNV was virulent to juvenile big-belly seahorses. Further studies will focus on the infection mechanism and preventive strategies for SHNNV.

\begin{abstract}
Abbreviations
BFNNV: barfin flounder NNV; CITES: Convention on International Trade in Endangered Species of Wild Fauna and Flora Conservation programs; CP: Capsid protein; CPE: cytopathic effects; DIG: digoxigenin; FBS: fetal bovine serum; FISH: Fluorescent in situ hybridization; GS: grouper spleen; IUCN: International Union for Conservation of Nature; NNV: Nervous necrosis virus; qRT-PCR: quantitative Real-Time PCR; RdRp: RNA-dependent RNA polymerase; RGNNV: Red-spotted grouper NNV; SHNNV: Seahorse nervous necrosis virus; SJNNV: striped jack NNV;
\end{abstract}


SSC: saline sodium citrate; TCID50: median tissue culture infective dose; TPNNV: tiger puffer NNV; VNN: viral nervous necrosis.

\section{Declarations}

\section{Ethics approval and consent to participate}

All animal experiments were conducted in accordance with the guidelines and with the approval of the Animal Research and Ethics Committees of Fisheries Research Institute of Fujian.

\section{Consent to publication}

Not applicable.

\section{Availability of data and materials}

Nucleotide and amino acid sequences supporting reported results can be found in the Additional file: File Supplementary material. All data are presented in the results section and expressed into graphic presentations. If any data, not already shared, are needed, they may be made available.

\section{Competing interests}

The authors declare that they have no competing interests.

\section{Funding}

This work was supported by the National Natural Science Foundation of China (41806151), Science and Technology Department of Fujian Province, project \#2014R1003-14, and Special Fund of Ocean and Fishery Development of Xiamen, project \#18CZY008HJ03.

\section{Authors' contributions}

XXC, JFQ, QW and LYZ conceived and designed the experiments; XXC and QW performed the experiments; XXC, JFQ, LBH and HYL analysed the data; JBL and FYQ contributed reagents/materials/analysis tools; XXC, QW and LYZ wrote the manuscript. All authors read and approved the final manuscript.

\section{Acknowledgements}

Not applicable.

\section{References}

1. Alves RmRN, Rosa IL. Seahorses in Traditional Medicines: A Global Overview. Springer

Berlin Heidelberg; 2013; DOI: 10.1007/978-3-642-29026-8_10. 
2. Vaidyanathan $T$, Vincent $A$. State of seahorse fisheries in India, nearly two decades after they were banned. Biodiversity and Conservation. 2021;30(5):1-31.

3. Aylesworth L, Phoonsawat, et al. . Effects of indiscriminate fisheries on a group of small data-poor species in Thailand. ICES Journal of Marine Science. 2018;75(2):642-652.

4. Vaidyanathan $T$, Zhang $X$, Balakrishnan $R$, Vincent $A$. Catch and trade bans for seahorses can be negated by non-selective fisheries. Aquatic Conservation: Marine and Freshwater Ecosystems. 2021;31:43-59.

5. CITES. Trade in seahorses. 2005; https://cites.org/eng/node/1780. Accessed 20 Sep, 2021.

6. Pollom R. Hippocampus abdominalis. The IUCN Red List of Threatened Species 2017: e.T10057A54903879. 2017; https://www.iucnredlist.org/species/10057/54903879. Accessed 20 Sep, 2021.

7. Nishizawa T, Furuhashi M, Nagai T, Nakai T, Muroga K, Asia SJAEM. Genomic classification of fish nodaviruses by molecular phylogenetic analysis of the coat protein gene. 1997;63(4):1633-1636.

8. Munday BL, Kwang J, Moody NJJoFD. Betanodavirus infections of teleost fish: a review. $2002 ; 25$.

9. Bandín I, Souto S. Betanodavirus and VER Disease: A 30-year Research Review. Pathogens. 2020;9(2).

10.Nakai BLMaT. Nodaviruses as pathogens in larval and juvenile marine finfish. World Journal of Microbiology \& Biotechnology. 1997;13(4):375-381.

11.Yoshikoshi K, Inoue K. Viral nervous necrosis in hatchery-reared larvae and juveniles of 
Japanese parrotfish, Oplegnathus fasciatus (Temminck \& Schlegel). Journal of Fish Diseases. 1990;13(1).

12.Bloch B, Gravningen K, Larsen JLJDoAO. Encephalomyelitis among turbot associated with picornavirus-like agent. 1991;10(1):65-70.

13.Renault T, Haffner P, Laurencien FB, Breuil G, Bonami JR. Mass mortalities in hatchery-reared sea bass (Lates calcarifer) larvae associated with the presence in the brain and retina of virus-like particles. Bull.eur.ass. fish Pathol. 1991;11(2):68-73.

14.Gomez D K SJ, Mushiake K, et al. PCR-based detection of betanodaviruses from cultured and wild marine fish with no clinical signs. Journal of Fish Diseases. 2004;27(10):603-608.

15.Panzarin V, Fusaro A, Monne I, et al. Molecular epidemiology and evolutionary dynamics of betanodavirus in southern Europe. Infection Genetics \& Evolution. 2012;12(1):63-70.

16. Olveira JG, Souto S, Dopazo CP, Bandín I. Isolation of betanodavirus from farmed turbot Psetta maxima showing no signs of viral encephalopathy and retinopathy. Aquaculture. 2013;406-407:125-130.

17.Iwamoto T, Okinaka Y, Mise K, et al. Identification of Host-Specificity Determinants in Betanodaviruses by Using Reassortants between Striped Jack Nervous Necrosis Virus and Sevenband Grouper Nervous Necrosis Virus. 2004;78(3):1256-1262.

18.Munday B, Huang B, Chang SF, et al. Determination of the complete nucleotide sequences of RNA1 and RNA2 from greasy grouper (Epinephelus tauvina) nervous necrosis virus, Singapore strain. 2001;82(3):647-653.

19.Shetty M, Maiti B, Santhosh KS, Venugopal MN, Karunasagar IJIJoV. Betanodavirus of 
Marine and Freshwater Fish: Distribution, Genomic Organization, Diagnosis and Control Measures. 2012;23(2):114-123.

20.Hall TAJNASS. BioEdit: A User-Friendly Biological Sequence Alignment Editor and Analysis Program for Windows 95/98/NT. 1999;41(41):95-98.

21.Tamura K, Stecher G, Peterson D, Filipski A, Kumar SJMB, Evolution. MEGA6: Molecular Evolutionary Genetics Analysis Version 6.0. 2013;30(12):2725-2729.

22.Ragoczy T, Bender MA, Telling A, Byron R, Groudine MJGD. The locus control region is required for association of the murine $\beta$-globin locus with engaged transcription factories during erythroid maturation. 2006;20(11).

23. Ho Y, Shewchuk BM, Liebhaber SA, Cooke NEJM, Biology C. Distinct chromatin configurations regulate the initiation and the maintenance of hGH gene expression. 2013;33(9):1723-1734.

24.Beliveau BJ, Boettiger AN, Avenda?O MS, et al. Single-molecule super-resolution imaging of chromosomes and in situ haplotype visualization using Oligopaint FISH probes. $2015 ; 6: 7147$.

25.Corp I. Released 2017. IBM SPSS Statistics for Windows, Version 25.0. Armonk, NY: IBM Corp. 2017; https://www.ibm.com/support/pages/node/417815. Accessed 20 Sep, 2021.

26. Glazebrook JS, Heasman MP, Beer SWDJJoFD. Picorna-like viral particles associated with mass mortalities in larval barramundi, Lates calcarifer Bloch. 1990;13(3):245-249.

27.Breuil G, Bonami JR, Pepin JF, Pichot Y. Viral infection (picorna-like virus) associated with mass mortalities in hatchery-reared sea-bass (Dicentrarchus labrax) larvae and juveniles. Aquaculture. 1991;97(2-3):109-116. 
28.Li L, He J, Mori KI, Nishioka T, Nakai TJFP. Mass mortalities associated with viral nervous necrosis in hatchery-reared groupers in the People's Republic of China. 2001;36(3):186-188.

29.Chi S, Shieh J, Lin SJDoAO. Genetic and antigenic analysis of betanodaviruses isolated from aquatic organisms in Taiwan. 2003;55(3):221-228.

30.Thiéry R, Cozien J, de Boisséson C, Kerbart-Boscher S, Névarez L. Genomic classification of new betanodavirus isolates by phylogenetic analysis of the coat protein gene suggests a low host-fish species specificity. J Gen Virol. 2004;85(Pt 10):3079-3087.

31.Chen N-C, Yoshimura M, Guan H-H, et al. Crystal Structures of a Piscine Betanodavirus: Mechanisms of Capsid Assembly and Viral Infection. PLoS pathogens. 2015;11(10):e1005203.

32.Valentina P, Anna T, Miriam A, et al. Molecular Basis for Antigenic Diversity of Genus Betanodavirus. PLoS ONE. 2016;11(7):e0158814.

33.Panzarin V, Cappellozza E, Mancin M, et al. In vitro study of the replication capacity of the RGNNV and the SJNNV betanodavirus genotypes and their natural reassortants in response to temperature. Vet Res. 2014;45:56.

34. Hata N, Okinaka Y, Iwamoto T, Kawato Y, Mori K-I, Nakai T. Identification of RNA regions that determine temperature sensitivities in betanodaviruses. Arch Virol. 2010;155(10):1597-1606.

35.Chi SC, Lo CF, Kou GH, Chang PS, Peng SEJJoFD. Mass mortalities associated with viral nervous necrosis (VNN) disease in two species of hatchery-reared grouper, Epinephelus fuscogutatus and Epinephelus akaara (Temminck \& Schlegel). 1997;20(3). 
36. Chi SC, Lo BJ, Lin SCJJoFD. Characterization of grouper nervous necrosis virus (GNNV). $2001 ; 24(1): 3-13$

37.Jaramillo D, Fielder S, Whittington RJ, Hick P. Host, agent and environment interactions affecting Nervous necrosis virus infection in Australian bass Macquaria novemaculeata. Journal of fish diseases. 2019;42(2):167-180.

38.Binesh CP. Elevation of temperature and crowding trigger acute viral nervous necrosis in zebra fish, Brachydanio rerio (Hamilton-Buchanan), subclinically infected with betanodavirus. Journal of fish diseases. 2014;37(3):279-282.

39. Thanasaksiri K, Sakai N, Yamashita H, Hirono I, Kondo H. Influence of temperature on Mx gene expression profiles and the protection of sevenband grouper, Epinephelus septemfasciatus, against red-spotted grouper nervous necrosis virus (RGNNV) infection after poly (I:C) injection. Fish \& shellfish immunology. 2014;40(2):441-445.

40.Ma H, Wen W, Su Y, et al. Epidemiological characterization of VNNV in hatchery-reared and wild marine fish on Hainan Island, China, and experimental infection of golden pompano (Trachinotus ovatus) juveniles. Arch Virol. 2015;160(12):2979-2989.

41.Nishizawa T, Gye HJ, Takami I, Oh M-J. Potentiality of a live vaccine with nervous necrosis virus (NNV) for sevenband grouper Epinephelus septemfasciatus at a low rearing temperature. Vaccine. 2012;30(6):1056-1063.

42.Ciulli S, Ostanello F, Battilani M, Prosperi S, Hedrick RP. Effect of temperature on Betanodavirus infection in SSN-1 cell line. Vet Res Commun. 2004;28 Suppl 1:283-286. 43.Ciulli S, Gallardi D, Scagliarini A, Battilani M, Hedrick RP, Prosperi S. Temperature-dependency of Betanodavirus infection in SSN-1 cell line. Diseases of 
aquatic organisms. 2006;68(3):261-265.

44.Bovo G, Nishizawa T, Maltese C, Borghesan F, Mas SD. Viral encephalopathy and retinopathy of farmed marine fish species in Italy. Virus Research. 1999;63(1-2):143-146.

45. Toffolo V, Negrisolo E, Maltese C, et al. Phylogeny of betanodaviruses and molecular evolution of their RNA polymerase and coat proteins. Mol Phylogenet Evol. 2007;43(1):298-308.

46.Mao M-G, Wen S-H, Perálvarez-Marín A, et al. Evidence for and characterization of nervous necrosis virus infection in Pacific cod (Gadus macrocephalus). Arch Virol. 2015;160(9):2237-2248.

\section{Supplementary material legends}

Sequence S1. Nucleotide sequence of SHNNV-cp gene.

Sequence S2. Amino acid sequence deduced from SHNNV-cp mRNA. 


\section{Supplementary Files}

This is a list of supplementary files associated with this preprint. Click to download.

- Supplementarymaterial.pdf 